\title{
quelques remarques sur les méthodes d'homogénéisation
}

\author{
some remarks on homogenization processes
}

\author{
J.L. AURIAUlT, D. CAILLERIE \\ Institut de Mécanique de Grenoble* \\ associé au CNRS
}

Rev. Franç. Géotech. n 49, pp. 43-50 (octobre 1989)

\section{Résumé}

De nombreux matériaux tels les composites, les poreux, etc. présentent une grande densité de fortes homogénéités fines. Les volumes considérés comprenant un très grand nombre d'hétérogénéités, la description n'est possible qu'en "moyenne» où le matériau est remplacé par un continu macroscopiquement équivalent: le matériau est alors dit homogénéisé.

De nombreuses méthodes d'homogénéisation sont disponibles. Elles peuvent se classer suivant qu'il existe des échelles caractéristiques séparées ou non, que le matériau est à structure périodique ou aléatoire. Nous présentons ici quelques indications sur ces différentes catégories en mettant l'accent sur leurs possibilités et leurs points communs. La méthode d'homogénéisation de structures périodiques est abordée plus en détail et des exemples sont présentés qui mettent en évidence ses possibilités.

\footnotetext{
Abstract

Numerous materials such as composites, porous media etc. exhibit a high density of small heterogeneities. Considering that the bulk volume of such materials is composed of a large number of heterogeneities, the only possible description is in the mean where the material is replaced by a macroscopically equivalent continuum: the material is said to be homogenized.

Available homogenization processes are numerous. They can be classified according to the existence of separated scales or not and to the material structure either periodic or random. We present some informations concerning the possibilities and the common features of the different sorts of processes. Focusing on the method of homogenization for periodic structures, some examples of application are presented which display its possibilities.
} 


\section{INTRODUCTION}

De nombreux matériaux présentent une forte densité d'hétérogénéités fines. Ce sont par exemple les composites, les sols, les poreux, les polycristaux, les suspensions, les mélanges de fluides séparés mais aussi la terre armée, l'ensemble sol-fondation sur pieux ou les structures réticulées dans la mesure où les armatures, les pieux, les poutres de la structure sont en grand nombre.

Lors de l'étude, il est difficile, voire impossible de prendre en compte les hétérogénéités une à une. Le problème s'avère en effet mal conditionné. La description de ces matériaux n'est possible qu'en « moyennex: l'idéal est de les remplacer par des continus macroscopiquement équivalents. On dit alors que les matériaux sont homogénéisés.

Dans la suite l'échelle microscopique ou locale sera l'échelle des hétérogénéités et l'échelle macroscopique l'échelle du matériau à étudier, contenant un grand nombre d'hétérogénéités.

La description macroscopique peut être obtenue soit par une étude phénoménologique ou expérimentale, directement à l'échelle macroscopique, soit par une technique d'homogénéisation, c'est-à-dire par passage de la description microscopique à la description macroscopique. C'est cette deuxième voie qui est abordée ici. Il n'est pas possible de donner un aperçu complet de toutes les techniques d'homogénéisation, fort nombreuses. Nous nous bornons donc principalement à la présentation de deux d'entre elles: lhomogénéisation de structures périodiques (HSP) (BENSOUSSAN et al., 1978; SANCHEZ-PALENCIA, 1980), et la modélisation statistique (MS) développée par KRÖNER (1986) pour les matériaux de structures aléatoires. D'une part ce sont sans doute les méthodes les plus efficaces, d'autre part certains liens de parenté entre elles permettent de tirer quelques idées générales.

La partie 2 présente des généralités sur les méthodes d'homogénéisation ainsi que les relations existant entre certaines d'entre elles. La modélisation statistique est ensuite présentée brièvement dans la troisième partie. En parallèle l'homogénéisation de structure périodique fait l'objet de la partie 4 , suivie dans la partie 5 d'une comparaison des possibilités de ces deux techniques. Enfin on donne quelques applications de la méthode HPS dans la dernière partie 6.

Cette présentation ne peut être exhaustive. Aussi nous passons sur certaines difficultés concernant les conditions d'application des méthodes d'homogénéisation, notamment celles qui sont liées aux conditions aux limites des matériaux étudiés.

\section{GÉNÉRALITÉS SUR LES MÉTHODES DE PASSAGE MICRO-MACRO}

Remarquons tout d'abord, et cela était sous-jacent dans l'introduction ci-dessus, que l'homogénéisation d'un milieu à forte densité d'hétérogénéités n'est pos- sible que si on considère des domaines contenant un grand nombre d'hétérogénéités. Si l est une dimension caractéristique des hétérogénéités et $\mathrm{L}$ une dimension caractéristique du volume de matériau ou du phénomène étudié, $\epsilon=1 / \mathrm{L}<<1$. Nous dirons alors que les échelles sont séparées. Deux grandes classes de matériaux peuvent alors être définies suivant que ces deux échelles sont séparées ou non. Pour la première, il n'existe pas d'autre échelle intermédiaire. L'homogénéisation est alors possible pour les matériaux caractérisés par une invariance (ou quasi-invariance) par translation à l'échelle microscopique, dans un sens qui sera précisé plus loin. Peuvent d'ailleurs coexister plus de deux échelles, mais à la condition qu'elles soient bien séparées les unes des autres. Et on passe alors de l'une à l'autre immédiatement supérieure par homogénéisation.

Lorsqu'il existe une infinité d'échelles non séparées (la deuxième classe), des résultats peuvent être obtenus lorsque la structure est invariante par dilatation. Par exemple dans le cas d'un réseau régulier de barres conductrices ou non conductrices réparties de façon aléatoire, la structure de la partie conductrice du réseau est invariante par dilatation lorsque la probabilité de barre dans l'état conducteur est voisine de la probabilité critique caractérisant le seuil conducteurnon conducteur du réseau. La théorie de la percolation (CLERC et al., 1983) permet alors, pour de telles structures, d'obtenir l'allure de la conductivité du réseau au voisinage du seuil.

Comme on le voit, il s'agit ici aussi d'homogénéisation puisqu'une propriété "moyenne», ici la conductivité effective du réseau est obtenue. Toutefois on réserve en général le terme d'homogénéisation aux techniques de passage micro-macro concernant les structures à échelles séparées. Nous n'abordons dans la suite que cette seule classe de matériaux.

\subsection{Méthodes d'homogénéisation à échelles séparées}

Donnons tout d'abord quelques exemples de matériaux hétérogènes en rapport avec le génie civil. L'échelle microscopique de longueur caractéristique I n'est "microscopique» qu'en comparaison de l'échelle macroscopique de longueur caractéristique L. Ainsi I peut prendre des valeurs qui n'ont rien de microscopique comparées à l'échelle humaine. Si pour les argiles I sera la taille des pores, par exemple $50 \AA$, un composite mat de verre-résine époxy aura un 1 $1 \mathrm{~mm}$, le béton un $1 \sim 1 \mathrm{~cm}$, une terre armée ou un talus drainé par géotextiles un $1 \sim 50 \mathrm{~cm}$, et enfin une fondation sur pieux un $1 \sim 5 \mathrm{~m}$. Sans qu'il soit possible de donner une frontière bien précise, on estime en général que l'homogénéisation devient performante pour $L>10$ l soit $\epsilon<0,1$.

Une autre caractéristique générale de toutes les méthodes est qu'elles utilisent des moyennes pour définir les grandeurs macroscopiques. Mais celles-ci sont soit introduites de façon arbitraire et ceci constitue un prérequis à l'échelle macroscopique qui impose ainsi la définition des grandeurs physiques macroscopiques, soit elles sont introduites par le processus de passage micro-macro et ceci est une garantie d'obtention de la "bonne" grandeur physique macroscopique. 
Comme on le voit sur les exemples ci-dessus deux classes de matériaux se dégagent à nouveau: les matériaux à structures périodiques et ceux à structures aléatoires. Les premiers sont étudiés par la méthode d'homogénéisation de structures périodiques (HSP) développée par BENSOUSSAN et al. (1978) et SANCHEZ-PALENCIA (1980) et maintenant appliquée à de nombreux domaines de la physique. Nous reviendrons en détail sur cette méthode dans la partie 4 et donnerons succinctement quelques exemples d'application dans la partie 6. Les seconds, c'est-àdire les matériaux à structures aléatoires font l'objet de très nombreuses techniques que nous ne pouvons pas rapporter toutes ici. Citons la méthode de Modélisation Statistique (MS) de KRÖNER (1986), les méthodes développées par GELHAR (1987) ou par MATHERON (1967), les méthodes auto-consistantes (cf. par exemple ZAOUI, 1987) et d'une façon générale les méthodes de moyennes proprement dites (GILBERT, 1987; HOWES et al., 1985; BEDFORD et al., 1983; NIGMATULIN, 1981), etc. La méthode introduite par KRÖNER (1986) sera présentée succinctement dans la partie 3. L'étude des matériaux à structures aléatoires présuppose l'hypothèse de stationnarité ou de quasi-stationnarité. Même si elle n'est pas toujours clairement énoncée, cette hypothèse est sous-jacente dans toutes les méthodes et nécessaire pour qu'une homogénéisation puisse être faite. Elle correspond à la propriété de périodicité des structures périodiques, présentant avec elle cette propriété d'invariance par translation, à opposer à l'invariance par auto-similitude utilisée pour les matériaux à échelles non séparées.

Il est intéressant de noter les points communs entre les méthodes d'homogénéisation les plus performantes. D'une part les méthodes auto-consistantes apparaissent comme équivalentes à la méthode MS dans le cas d'un désordre parfait (KRÖNER, 1972, 1986). D'autre part, pour un composite élastique, les coefficients d'élasticité effectifs (macroscopiques) ont une écriture formelle identique, qu'ils soient obtenus par la méthode MS pour un composite aléatoire ou par la méthode HPS pour un composite périodique (KRÖNER, 1980).

Pour les problèmes de filtration, il est aisé de constater qu'un résultat du même ordre est valable pour un poreux composite, en comparant les écritures formelles du coefficient de perméabilité effectif obtenu par MATHERON (1967) et celui obtenu par la méthode HPS.

Mais d'une façon générale, plus la séparation des échelles est propre, meilleurs sont les résultats. Cela donne un avantage décisif à la méthode HSP. La périodicité introduit en effet une séparation des échelles parfaites, I pouvant être définie comme dimension caractéristique de la période. Ceci n'est pas possible pour les matériaux à structure aléatoire.

\subsection{La méthode d'homogénéisation idéale}

La méthode d'omogénéisation idéale prétend, à partir de la description complète à l'échelle microscopique, découvrir la description complète à l'échelle macroscopique. Ainsi à l'échelle microscopique sont supposées connues:

- les équations de conservation;

- les rhéologies avec la valeur des parametres:

- les grandeurs physiques qui décrivent les phénomènes étudiés:

- la géométrie.

Et le processus de passage micro-macro doit donner:

- les équations de conservation;

- les rhéologies et les paramètres effectifs;

- les bonnes grandeurs physiques qui décrivent les phénomènes à l'échelle macroscopique.

Bref le processus idéal doit être indépendant de toute hypothèse à l'échelle macroscopique afin de garantir la qualité scientifique du résultat. Quant on aura remarqué que les structures des modélisations macroscopiques peuvent être très différentes des structures des modélisations microscopiques correspondantes, on comprendra l'importance de ce point; un exemple en est la filtration dans un poreux rigide: à l'échelle des pores la description pour un fluide newtonien est celle de STOKES avec la rhéologie correspondante; à l'échelle macroscopique la modélisation est celle de la loi de DARCY, certes aussi l'expression du principe fondamental de la mécanique, mais avec une structure fort différente où la rhéologie se présente maintenant intimement mélangée à la dynamique (AURIAULT, 1986).

Le processus idéal doit aussi permettre la localisation, c'est-à-dire la détermination des champs de grandeurs physiques locales à partir des valeurs des grandeurs physiques macroscopiques. Il est alors évident que la seule méthode qui réponde à ces conditions est la méthode HSP puisque la périodicité assure la description complète du matériau. Par contre il n'est jamais possible de décrire totalement un matériau aléatoire (sauf dans le cas limite du désordre parfait). Ainsi les méthodes associées aux structures aléatoires ne peuvent pas être parfaites. La description locale étant incomplète, il est nécessaire d'introduire à l'échelle macroscopique des prérequis sur le danger desquels nous avons attiré l'attention plus haut.

\section{LA MODÉLISATION STATISTIQUE}

Cette méthode (KRÖNER, 1986) est principalement utilisée pour obtenir des informations sur les coefficients effectifs d'un composite élastique soumis à une sollicitation statique.

\section{Les hypothèses sont:}

- les échelles sont séparées: $\varepsilon=1 / L<<1$. Cette hypothèse est sous-jacente mais il n'en est pas fait usage systématique dans le passage micro-macro:

- le matériau a une structure aléatoire;

- le milieu stationnaire est de dimension infinie. L'hypothèse d'ergodicité est admise: la moyenne d'ensemble égale la moyenne de volume; 
- un prérequis est imposé: $\langle\sigma \underline{\epsilon}\rangle=\left\langle\sigma{ }^{\prime}\right\rangle\langle\underline{\epsilon}\rangle$ où l'opérateur $\langle$.$\rangle représeñte la moyeñne de vo-$ lume, $\sigma$ la contrainte et $\epsilon$ la déformation. Ceci implique que:

- la contrainte macroscopique est $\langle\underline{\sigma}\rangle$;

- la déformation macroscopique est $\langle\underset{\sim}{\epsilon}\rangle$;

- le matériau a un comportement macroscopique élastique;

- la moyenne de la densité locale d'énergie élastique égale la densité macroscopique d'énergie élastique.

Comme nous l'avons indiqué plus haut la description locale d'un matériau à structure aléatoire n'est jamais parfaitement connue. L'intérêt de la modélisation statistique de KRÖNER est de parfaitement s'adapter à cette situation: la méthode fournit des bornes supérieures et inférieures pour les coefficients effectifs, ces bornes étant d'autant plus rapprochées que l'information augmente.

La méthode peut être présentée à partir de la formulation intégrale du problème aux limites pour un milieu infini, prenant en compte toutes les hétérogénéités et utilisant la fonction de GREEN modifiée en déformation. Comme cette fonction de GREEN n'est évidemment pas connue, compte tenu de la complexité du problème, KRÖNER (1986) introduit la fonction de GREEN modifiée en déformation associée à un milieu élastique fictif homogène. Cette fonction est parfaitement connue. KRÖNER montre alors que la vraie fonction de GREEN s'exprime à partir de cette fonction de GREEN fictive et de la répartition c du tenseur élastique. Puis, utilisant la définition suivante du tenseur élastique effectif $C_{\text {eff }}$ qui découle du prérequis mentionné ci-dessus:

$$
\langle\underline{\sim}\rangle=\left\langle\underline{\sim}_{\underset{\epsilon}{\epsilon}}\right\rangle=\underline{\sim}_{\text {eff }}\langle\underline{\sim}\rangle \text {, }
$$

$C_{\text {eff }}$ est représenté sous forme d'un développement eñ série relativement aux fonctions de corrélation d'ordre croissant de c.

Des considérations énergétiques permettent ensuite de montrer que la série tronquée, donc ne nécessitant que la connaissance des fonctions de corrélation d'ordre inférieur à un ordre donné n correspond à

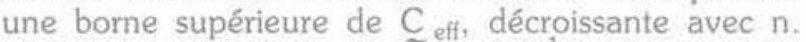
La même approche avec $\mathrm{s}^{2}=c^{-1}$ conduit symétriquement à une suite croissãnte de bornes inférieures. Les bornes les moins précises sont celles de VOIGT, $\langle\mathrm{c}\rangle$ et REUSS, $\left\langle\mathrm{c}^{-1}\right\rangle^{-1}$. Elles ne nécessitent que la connaissance dẽs moyennes de $\mathrm{c}$ et $\mathrm{s}$ et sont alors les meilleures possibles. L'introduction de fonctions de corrélation d'ordre 2 conduit à des bornes plus rapprochées. Si le matériau est macroscopiquement isotrope et les modules élastiques de deux grains voisins non corrélés, ce sont les bornes d'HASHIN et al., (1963) ; et ainsi de suite. La connaissance de toutes les fonctions de corrélation conduit en théorie au coefficient effectif lui-même.

\section{HOMOGÉNÉISATION DE STRUCTURES PÉRIODIQUES}

On trouvera plus de détails sur cette technique en BENSOUSSAN et al. (1978) et SANCHEZ-PALENCIA (1980).
Les hypothèses de départ sont plus réduites que dans la méthode décrite dans le paragraphe précédent: - les échelles sont séparées: $\epsilon=1 / L<<1$. Mais ici il est fait un usage systématique de l'existence d'un tel petit paramètre:

- en introduisant en plus de la variable d'espace classique dite variable lente, une variable d'espace dilatée $y=x / \epsilon$ ou variable rapide où l'échelle est bien apprõpriéé à la description des hétérogénéités fines. La variable lente $x$ sert à décrire le comportement macroscopique équivalent ;

- en recherchant les inconnus $\varnothing$ sous la forme de développements asymptotiques en puissance de $\varepsilon$;

- le milieu est finement périodique. La structure périodique du matériau induit certaines propriétés de périodicité des inconnues. Ainsi un fluide filtrant dans un poreux périodique sera caractérisé par un champ de vitesse périodique. D'autre part la période, dont les dimensions sont $O$ (1), peut varier lentement dans l'espace c'est-à-dire de $O$ (1) sur les distances $O$ (L). De même les inconnues, qui ont des fluctuations locales dues aux hétérogénéités et bien décrites par la variable $y$, sont aussi variables à l'échelle macrosco. pique, dũ fait de la quasi-périodicité éventuelle évoquée ci-dessus ou des conditions aux limites macroscopiques qui induisent des gradients à cette échelle. La variable $x$ décrit ces variations. Ainsi les inconnues peuvent êtrê cherchées sous la forme:

$\varnothing=\varnothing^{(0)}(\underline{x}, \underline{y})+\varnothing^{(1)}(\underline{x}, \underline{y})+\epsilon^{2} \varnothing^{(2)}(\underset{\sim}{x}, \underline{y})+\ldots$ où les $\varnothing^{\prime}$ sont périodiques en $\underline{y}$.

Le comportement macroscopique équivalent est obtenu pour $\varepsilon$ tendant vers zéro et fait donc intervenir les premiers termes non nuls de tels développements. D'autres approches équivalentes sont possibles que nous ne présentons pas ici, les fondements étant identiques.

La méthode présente toutes les caractéristiques de la méthode idéale évoquée plus haut. Ceci découle principalement du fait que la périodicité permet une connaissance parfaite de la description locale. Ainsi un problème aux limites concernant un matériau périodique comportant un grand nombre d'hétérogénéités s'étudie de la façon suivante au moyen de la méthode HSP:

a. Introduisant pour les inconnues des développements asymptotiques du type présenté ci-dessus dans les équations traduisant la description locale, on obtient pour les différentes puissances de $\varepsilon$ une suite de problèmes aux limites à résoudre sur une période. L'étude de ces problèmes introduit naturellement la notion de moyenne de volume, les grandeurs macroscopiques à prendre en compte, les équations vérifiées par ces grandeurs. On obtient ainsi la description macroscopique équivalente: équations de bilans, rhéologies, coefficients effectifs mais aussi la correspondance entre les grandeurs moyennes et les champs locaux, c'est-à-dire la localisation. Le sens physique des grandeurs moyennes doit faire l'objet d'une étude particulière. En effet des grandeurs comme la contrainte ou la vitesse, qui sont des flux, n'ont de sens physique macroscopiquement que définies par des moyennes de surface. La relation entre moyennes de volume et de surface, permise par la méthode, est donc nécessaire à une bonne écriture de la description macroscopique. 
b. Les matériaux étudiés sont généralement de dimensions finies. La périodicité est rompue près de ces frontières macroscopiques et n'y subsiste que tangentiellement. La solution présentée en a n'est valable que très loin (mesurée en variable $y$ ) de ces frontières. Il convient donc d'introduire le Tong de cellesci des couches limites de raccordement de la solution homogénéisée obtenue en a avec les conditions aux frontières macroscopiques. Cela permet de définir dans le même temps le type de conditions aux limites correspondant au problème étudié et d'obtenir les champs locaux au niveau de ces couches limites (cf. LEVY et al., 1975, 1977, pour deux études particulières concernant les poreux et SANCHEZPALENCIA, 1987 pour des remarques plus générales).

c. Le problème aux limites macroscopiques est résolu, utilisant la description macroscopique fournie par l'homogénéisation et les bonnes conditions aux limites induites par l'étude des couches limites.

d. Localisation. Les champs locaux sont donnés dans la masse par la solution en milieu infini de l'homogénéisation (cf. a) et près des frontières macroscopiques par l'étude des couches limites (cf. b). Remarquons que cette dernière étude conduit quelquefois à des effets particuliers de surface où il n'est pas possible d'obtenir une description macroscopique équivalente (AURIAULT et al., 1987).

\section{COMPARAISON DES MÉTHODES HSP ET MS}

Rappelons tout d'abord les points communs. Les deux méthodes s'adressent à des matériaux à échelles séparées: il existe un petit paramètre $\epsilon=1 / \mathrm{L}<<1$. Bien entendu la non-utilisation systématique de ce fait dans la méthode MS explique certaines faiblesses de celle-ci que nous notons dans la suite. La même écriture formelle des coefficients effectifs d'un composite élastique montre que les deux méthodes sont voisines et on peut supposer que cette propriété s'étend à tout problème elliptique. L'invariance par translation (périodicité ou stationnarité) représente un troisième point commun. Enfin, mais là le résultat est obtenu par des voies très différentes, les deux méthodes permettent d'étudier le sens physique des moyennes de volume. La solution découle de l'hypothèse d'ergodicité pour la méthode MS, de la périodicité et du caractère solenoïdal local des grandeurs pour la méthode HSP (cf. AURIAULT, 1986 pour des exemples simples). La méthode HSP permet toutefois d'envisager des exemples où la moyenne de volume n'égale pas la moyenne de surface. On consultera par exemple LEVY (1981) ou AURIAULT (1987), AURIAULT et al. (1989).

Les différences concernent d'abord le problème des prérequis à l'échelle macroscopique. Ceux-ci qui imposent a priori des restrictions sur la description macroscopique, privent de généralité la théorie MS ainsi que la plupart des méthodes d'homogénéisation, excepté la méthode HSP. Et il est indéniable qu'ils ne favorisent pas la découverte de descriptions «exotiques», c'est-à-dire des descriptions qui n'utilisent pas les règles phénoménologiques macroscopiques habituelles.

La cohérence physique des résultats est un point très important. Si nous appliquons la méthode HSP telle qu'elle est décrite au paragraphe 4. la description macroscopique est obtenue au moyen d'équations de bilan, de lois rhéologiques, avec les grandeurs macroscopiques adéquates. Si le sens physique de ces dernières doit être étudié comme nous venons de le mentionner, il est nécessaire aussi de vérifier les cohérences de type énergétique. Ainsi pour un composite élastique sous sollicitation statique, la moyenne de la densité d'énergie élastique locale doit égaler la densité d'énergie élastique macroscopique définie à partir des bonnes grandeurs physiques macroscopiques. La méthode HSP se prête bien à cette étude. En ce qui concerne la méthode MS, la question ne se pose pas puisque la cohérence est incluse dans le prérequis.

La localisation est un autre avantage de la méthode HSP que ce soit pour obtenir les champs locaux dans la masse du matériau ou pour se libérer du cas simpliste que représente le milieu infini. L'introduction des couches limites de raccordement permet à la méthode HSP de traiter des problèmes aux limites sur des domaines finis.

L'utilisation systématique du petit paramètre $\varepsilon$ donne à la méthode HSP un avantage décisif. Ainsi il n'est pas possible avec la méthode MS d'envisager la dynamique d'un composite élastique ou l'introduction de forces de volume (KRÖNER, 1986). Par contre, la méthode HSP montre rapidement que la rhéologie macroscopique obtenue en statique est utilisable en description dynamique macroscopique, du moins pour les cas classiques (cf. AURIAULT et al., 1985). D'autres exemples résident dans la possibilité de traiter avec la méthode HSP les problèmes à plusieurs échelles séparées ainsi que les problèmes où, en plus du petit paramètre d'homogénéisation $\varepsilon$, d'autres petits paramètres interviennent concernant la géométrie interne de la période ou les coefficients rhéologiques des matériaux composites. Ainsi de nombreuses situations peuvent être étudiées suivant les niveaux respectifs des différents petits paramètres. On trouvera dans le paragraphe suivant quelques exemples de telles applications.

La méthode HSP permet de résoudre rapidement le problème de savoir si la mesure ponctuelle d'une grandeur physique $\varnothing$ est licite ou non au sein d'un matériau hétérogène. En fait le comportement est donné au premier ordre par $\varnothing^{(0)}(x, y)$ et tout le problème se ramène à celui de savơir sí la grandeur macroscopique $\left\langle\varnothing^{(0)}\right\rangle$ égale en tout point de la période la grandeur $\varnothing^{(0)}(x, y)$. Ceci n'est possible que si $\varnothing^{(0)}(x, y)$ est indêpendant de la variable $y$. C'est le cas pãr exemple pour la température $T$ dãns les problèmes de transferts thermiques dans un composite de matériaux à conductivités de même ordre de grandeur au regard de $\epsilon$ : $T^{(0)}$ est fonction de la variable lente $\mathrm{x}$ seule et la mesure ponctuelle est licite (AURIAULT, 1983). Dans le problème de filtration d'un liquide newtonien dans un poreux, la 
pression est telle que $\mathrm{p}^{(0)}=\mathrm{p}^{(0)}(\mathrm{x})$ alors que la vitesse (évidemment) dépend de la variable rapide $y$ : $v^{(0)}=v^{(0)}(x, y)$ (AURIAULT, 1986). La mesưre ponctuelle est licite pour la pression alors que la mesure de la vitesse doit être globale (une moyenne de surface !). De telles conclusions sont aussi possibles pour les autres méthodes d'homogénéisation et les raisonnements reposent plus ou moins sur la présence d'échelles séparées. On consultera à ce sujet WHITAKER (1986), NOZAD et al. (1985).

Signalons aussi le problème du choix du volume élémentaire représentatif (VER) pour certaines techniques de moyenne. Alors que pour la méthode HSP le problème ne se pose pas car le VER est la période, le VER est nécessairement approché pour les matériaux aléatoires. Si sa taille est $\mathrm{O}(1)$, il s'avère que pour un même matériau, le VER optimal dépend de la grandeur étudiée ainsi que de la valeur de cette grandeur. Pour un poreux de VER optimal pour obtenir la porosité diffère du VER optimal pour la perméabilité. Ceci est la conséquence de la mauvaise séparation des échelles. Une bonne étude concernant les poreux non saturés se trouve en ROUGER (1988).

Enfin la question se pose de savoir s'il est possible de transférer quelques avantages de la méthode d'homogénéisation de milieux périodiques à l'étude des matériaux aléatoires. Il semble que pour de nombreux phénomènes et milieux aléatoires finement hétérogènes, la structure de la description macroscopique soit celle obtenue par la méthode HSP en pratiquant donc ce processus sur un matériau périodique fictif de "même nature». Les coefficients effectifs sont ensuite à déterminer par une autre méthode. Une tentative timide utilisant la périodisation du matériau aléatoire est aussi présentée par CHEREL et al. (1987).

\section{QUELQUES EXEMPLES D'APPLICATION DE LA MÉTHODE D'HOMOGÉNÉISATION DE STRUCTURE PÉRIODIQUE}

Nous avons classé les quelques exemples ci-dessous suivant qu'ils font appel pour leurs études:

- au seul petit paramètre $\epsilon$, donc à des milieux avec seulement deux échelles séparées;

- à plusieurs petits paramètres $\epsilon$, donc à avec plusieurs échelles séparées;

- enfin à plusieurs petits paramètres $\epsilon, \gamma, \nu$ etc., où $\epsilon$ est le petit paramètre d'homogénéisation et $\gamma, \nu$ etc. d'autres petits paramètres introduits par la géométrie. les propriétés des matériaux, etc. En fait cette classification n'est pas rigoureuse et certains exemples pré. sentés dans la première catégorie relèvent aussi bien de la dernière.

\subsection{Un seul petit paramètre}

L'exemple le plus simple concerne l'étude des composites élastiques (DUVAUT, 1976). Les comportements microscopiques et macroscopiques possèdent la même structure de description. Il s'agit-là du problème abordé aussi par la méthode MS. L'étude des composites à l'état plastique est un autre exemple, moins simple (DE BUHAN et al., 1987; DE BUHAN, 1986; PASTOR et al., 1986; SUQUET, 1987). Notons pour les exemples ci-dessus, la grande importance de l'effet de bord (SANCHEZ-PALENCIA, 1987; AURIAULT et al., 1987; TURGEMAN et al., 1987).

L'étude de la filtration dans un poreux rigide est un exemple où les structures des descriptions microscopiques et macroscopiques sont très différentes, même si elles représentent dans les deux échelles, équations de NAVIER-STOKES et loi de DARCY, l'écriture du principe fondamental de la mécanique (ENE et al., 1975; AURIAULT, 1986). Le cas de sollicitations dynamiques (LEVY, 1979; AURIAULT, 1986) présente la particularité que la loi macroscopique de filtration ou loi de DARCY généralisée est une loi « exotique» de dynamique héréditaire: le fluide filtrant se souvient de ses accélérations passées.

L'étude des suspensions dans un liquide newtonien (FLEURY, 1980; LEVY et al., 1983; SANCHEZ. PALENCIA, 1985) ainsi que des mélanges de fluides (LEVY, 1981: AURIAULT et al., 1989) permet comme dans létude de tout mélange de mettre clairement en évidence les causes microscopiques de comportements macroscopiques mono ou pluriphasiques. Nous verrons en 6.3 un autre exemple concernant le poreux saturé.

\subsection{Plusieurs échelles séparées}

On peut ranger dans cette catégorie les poreux rigides à double porosité (LEVY, 1988). Trois échelles bien séparées sont utilisées. Une première échelle est celle des pores où la description relève des équations de STOCKES. La deuxième échelle est celle des fissures. A cette échelle les équations de STOCKES sont valables dans les fissures et le poreux équivalent au microporeux de l'échelle précédente est décrit par la loi de DARCY. Enfin à l'échelle macroscopique l'homogénéisation conduit à une loi de DARCY. De nombreux problèmes concernant de tels milieux restent ouverts. En particulier l'homogénéisation devrait permettre de valider ou d'invalider certaines descriptions macroscopiques introduites directement à cette échelle par des études phénoménologiques, par exemples celles de BARENBLATT et al. (1960) ou de WARREN et al. (1963)

Le poreux rigide à une échelle microscopique (celle des pores) et deux échelles macroscopiques séparées (celle d'un milieu poreux équivalent inhomogène et celle du milieu poreux macroscopiquement équivalent à ce dernier) est un autre exemple. On passe ainsi d'une description utilisant les équations de STOKES à l'échelle microscopique à deux descriptions utilisant la loi de DARCY aux deux échelles macroscopiques.

\subsection{Plusieurs petits paramètres}

Les problèmes faisant intervenir plusieurs petits paramètres ne peuvent être abordés que par une méthode 
d'homogénéisation utilisant systématiquement le moyen de mesure que représente le petit paramètre $\varepsilon$. En effet, les ordres relatifs de grandeur de ces paramètres sont un point clef de l'étude et gouvernent la structure de la description macroscopique. L'exemple type est représenté par le poreux élastique rempli de liquide visqueux newtonien (AURIAULT, 1986). Suivant la valeur de la viscosité (en fait de la contrainte visqueuse comparée à la pression et à la contrainte dans la matrice, relativement au petit paramètre $\varepsilon$ ), le comportement macroscopique peut être très différent. Pour une "faible» viscosité, la description macroscopique est une description diphasique «à la BIOT "; pour une viscosité «moyenne » la description est celle d'un milieu monophasique élastique; et pour une «forte* viscosité la description est celle d'un milieu monophasique visco-élastique.

La filtration dans un poreux classique (où la géométrie ne fait pas intervenir d'autre petit paramètre) est régie par la loi de DARCY; quand la matrice est constituée de particules (où de fibres minces) petites par rapport à la période, ce qui introduit un petit paramètre géométrique supplémentaire, la description devient celle d'une loi de BRINKMAN (LEVY, 1983). Dans le même ordre d'idée, on trouvera en CIORANESCU et al. (1985), l'étude du problème de DIRICHLET pour un domaine avec de petits trous.

Les talus drainés par des nappes de géotextiles (AURIAULT et al., 1982) introduisent outre $\varepsilon$ deux autres petits paramètres caractérisant l'un la géométrie de la période (rapport des épaisseurs de géotextile et de sol). lautre les propriétés hydrauliques (rapport des perméabilités du sol et du géotextile). L'étude de la terre armée (PASTOR et al., 1986), comme la diffusion stationnaire dans un corps contenant de petites inclusions de grande conductivité (CAILLERIE, 1983), le comportement de corps élastiques renforcé de fibres minces de grande rigidité (CAILLERIE, 1981) ou la statique des plaques minces élastiques à structure fine périodique (CAILLERIE, 1982) sont aussi des problèmes à trois petits paramètres.

Citons enfin l'homogénéisation de structures réticulées qui permet de remplacer ces structures par un continu équivalent (CIORANESCU et al., 1986, 1988). $\mathrm{Ce}$ type de problème introduit outre $\varepsilon$, un deuxième petit paramètre caractérisant la géométrie de la période.

\section{CONCLUSION}

Les quelques exemples présentés ci-dessus indiquent clairement que la grande efficacité de la méthode d'homogénéisation de structures périodiques est la conséquence de la propriété de périodicité quí permet une description complète de l'échelle locale, mais aussi de l'utilisation systématique du petit paramètre d'échelles séparées. Celui-ci constitue une jauge pour mesurer l'importance relative des phénomènes physiques et des caractéristiques physiques et géométriques des milieux hétérogènes étudiés.

\section{BIBLIOGRAPHIE}

Cette bibliographie ne saurait être extensive.
AURIAULT J.-L. (1983), Effective macroscopic description for heat conduction in periodic composites. J. Heat Mass Transfer, 26, 6, pp. 861-869.

AURIAULT J.-L. (1986), Mécanique des milieux poreux saturés déformables. Cours de $3^{e}$ cycle MMGE, Grenoble, pp. 1-71.

AURIAULT J.-L. (1987), Non saturated deformable porous media: quasi-statics. Transport in Porous Media, 2, 1, pp. 45-64.

AURIAULT J.-L., BONNET G. (1987), Surface effects in composite materials: two simple exam ples. Int. J. Engng. Sci., 25, 3, pp. 307-323.

AURIAULT J,-L., BORNE L., CORDARY D. (1982), Consolidation des remblais au moven de textiles: étude par homogénéisation. CR, $2^{e}$ Congrès Int. des Géotextiles, Las Végas, août 1982. pp. 31-36.

AURIAULT J.-L., BONNET G. (1985), Dynamique des composites élastiques périodiques. Arch. Mech., 37, 4-5, pp. 269-284.

AURIAULT J.-L., BONNET G., LEBAIGUE $O$ (1989), Dynamics of two immiscible fluids flowing through deformable porous media. Transport in Porous Media, 4, pp. 105-128.

AURIAULT J.-L., LEBAIGUE O. (1989), Acoustic waves in a mixture of fluids with capillary effects. A paraître, Int. J. Engng. Sci.

BARRENBLATT G.I., ZHELTOV Y.P., KOCHINA I.N. (1960), Basic concepts in theory of seepage of homogeneous liquids in fissured rocks. PMM, 24,5 , pp. 852-864.

BEDFORD A., DRUMHELLER D.S. (1983), Recent advances. Theories of immiscible and structured mixtures. Int. J. Engng. Sci., 21. 8. pp. 863-960.

BENSOUSSAN A., LIONS J.L., PAPANICOLAOU G. (1978). Asymptotic analysis for periodic structu. res. North-Holland Publishing Company, Amsterdam

DE BUHAN P. (1986), Approche fondamentale du calcul de la rupture. Thèse de Doctorat d'Etat, Paris V1, 24 octobre 1986.

DE BUHAN P., SALENCCON J. (1987). Analyse de la stabilité des ouvrages en sols renforcés par une méthode d'homogénéisation. Revue Française de Géotechnique, 41, pp. 29-43.

CAILLERIE D. (1981), Homogénéisation d'un corps élastique renforcé par des fibres minces de grande rigidité réparties périodiquement. CRAS Paris, série II, t. 292, 9 février 1981, pp. 477-480.

CAILLERIE D. (1982), Etude de quelques problèmes de perturbation en théorie de l'élasticité et de la conduction thermique. Thèse de Doctorat d'Etat, Paris VI, 26 avril 1982.

CAILLERIE D. (1983), Etude de la conduction stationnaire dans un domaine comportant une répartition périodique d'inclusions minces de grande conductivité. RAIRO, Analyse numérique/Numerical Analysis, 17, 2, pp. 137-159.

CHEREL L., BONNET G., AURIAULT J.-L. (1987), Homogenization by local periodization. VIe con- 
grès Franco-Polonais de mécanique non linéaire, Villard-de-Lans, 28 septembre-1 $1^{\text {er }}$ octobre 1987. A paraitre Arc. Mech.

CIORANESCU D., MURAT F. (1985), Un terme étrange venu d'ailleurs. Rapport $\mathrm{n}^{\circ} 82005$, Laboratoire d'Analyse Numérique, Université Pierre et Marie Curie, Paris.

CIORANESCU D., SAINT-JEAN-PAULIN J. (1986), Reinforced and honeycomb structures. J. Math. Pures et Appliquées, 65, pp. 403-422.

CIORANESCU D., SAINT-JEAN-PAULIN J. (1988), Elastic behaviour of very thin cellular structures dans Material instabilities and continuum mechanics and related problems». Ed. J.M. BALL, Oxford Science Publications, Clarendon Press Oxford, 1988, pp. 65-75.

CLERC J.P. et al. (1983), La percolation. Annales de Physiques, 8, 1, pp. 4-105.

DUVAULT G. (1976), Analyse fonctionnelle et mécanique des milieux continus. Application à l'étude des matériaux composites élastiques à structure périodique - Homogénéisation, dans «Theorical and Applied Mechanics». W.T. KOITER ed., North Holland, pp. 119-132.

ENE H.I., SANCHEZ-PALENCIA E. (1975), Equations et phénomènes de surface pour l'écoulement dans un modèle de milieu poreux. Journal de Mécanique, 14, 1, pp. 73-108.

FLEURY F. (1980), Propagation of waves in a suspension of solid particles. Wave Notion, 2, pp. $39-50$.

GELHAR L.W. (1987), Stochastic analysis of solute transport in saturated and unsaturated porous media, in "Fundamentals of Transport Phenomena in Porous Media», ed. J. BEAR et Y. CORAPCIOGLU, Martinus NIJHOFF Publishers, pp. $657-700$.

GILBERT F. (1987), Description des sols saturés par une méthode d'homogénéisation dans «Manuel de rhéologie des géomatériaux». Presses des Ponts-et-Chaussées, pp, 317-333.

HASHIN Z., SHTRIKMAN S. (1963), A variational approach to the theory of the elastic behaviour of multiphase materials. J. Mech. Phys. Solids, 11, pp. 127-140.

HOWES F., WHITAKERS. (1985), The spacial averaging theorem revisited. Chem. Engng. Sci., 40 , 8, pp. 1387-1392.

KRÖNER E. (1972), Statistical continuum mechanics. Springer Verlag WIEN.

KRÖNER E. (1980), Effective elastic moduli of periodic and random media: a unification. Mechanics Research Communications, 7, 5, pp. 323-327.

KRÖNER E. (1986), Statistical modelling in «Modelling small deformations of polycrystals . Chap. 8, J. GITTUS, J. ZARKA eds, Elsevier Appl. Sci. Publ., London.

LEVY T. (1981), Milieu élastique soumis à des forces volumiques de répartition périodique fine. Journal de Mécanique, 20, 3, pp. 497-508.

LEVY T. (1981), Propagation of waves in a mixture of fluids. Int. J. Engng. Sci., 19, pp. 83-90.
LEVY T. (1983), Fluid flow through an array of fixed particles. Int. J. Engng. Sci., 21, 1, pp. 11-23.

LEVY T. (1988), Ecoulement d'un fluide dans un milieu poreux fissuré. CRAS, Paris, t. 306, série II, pp. 1413-1417.

LEVY T., SANCHEZ-PALENCIA E. (1975), On Bounday conditions for fluid flow in porous media. Int. J. Engng. Sci., 13, pp. 923-940.

LEVY T., SANCHEZ-PALENCIA E. (1977), Equations and interface conditions for acoustic phenomena in porous media. J. of Mathematical Analysis and Application, 61, 3, pp. 813-834.

LEVY T., SANCHEZ-PALENCIA E. (1983), Suspension of solid particles in a newtonian fluid. J. of Non-newtonian Fluid Mechanics, 13, pp. 63-78.

MATHERON G. (1967), Eléments pour une théorie des milieux poreux. Masson et Cie.

NIGMATULIN R.1. (1981), Three-dimensional averaging in the mechanics of heterogeneous media. Fluid Mechanics, 10, 4, pp. 72-107.

NOZAD I., CARBONEL R.G., WHITAKER S. (1985), Heat conduction in multiphase systems. 1. Theory and experiment for two phase systems. Chem. Engn. Sci., 40, 5, pp. 843-855.

PASTOR J., TURGMAN S., CISSS A. (1986), Calculation of limit loads of structures in soils with metal reinforcement. Proc. European Conference on Numerical Methods in Geomechanics. Stuttgart.

ROUGER P. (1988), Séchage des poreux granulaires par thermomigration: analyse théorique et résultats expérimentaux. Thèse de Doctorat, Grenoble, 29 novembre 1988 .

SANCHEZ-PALENCIA E. (1980), Non-homogeneous media and vibration theory. Lecture Notes in Physics, 127, Springer-Verlag Berlin.

SANCHEZ-PALENCIA E. (1985), Current problems in high concentration suspensions. J. de Mécanique Th. et Appl., n ${ }^{\circ}$ spécial, pp. 21-51.

SANCHEZ-PALENCIA E. (1987), Boundary layers and edge effects in composites, dans Lectures Notes in Physics 272 «Homogenization technics for composite median, Springer-Verlag Berlin, pp. 122-192.

SUQUET P. (1987), Elements of homogenization for inelastic solid mechanics in "Homogenization techniques for composite media». Lecture Notes in Physics $n^{\circ} 272$, Springer-Verlag, pp. 193-278.

TURGEMAN S., PASTOR J. (1987), Comparaison des charges limites d'une structure hétérogène et homogénéisée. Journal de Mec. Th. et Appl., 6, 1, pp. 121-143.

WARREN J.O., ROOT P.J. (1963), The behaviour of naturally fractured reservoirs. Soc. Petrol. Eng. J., pp. 245-255.

WHITAKER S. (1986), Flow in porous media. I. A theorical derivation of Darcy's law. Transport in Porous Media, 1, pp. 3-25.

ZAOUI A. (1987), Approximate statistical modelling and applications, dans Lecture Notes in Physics $n^{\circ} 272$, «Homogenization techniques for composite media». Springer-Verlag Berlin, pp. 338-397. 